\title{
Investigation of the direct DNA damages irradiated by protons of different energies using geant4-DNA toolkit
}

\author{
Z. Ahmadi Ganjeh ${ }^{1}$, M. Eslami-Kalantari ${ }^{*}$, M. Ebrahimi Loushab ${ }^{2}$, \\ A.A. Mowlavi3,4 \\ ${ }^{1}$ Department of Physics, School of Science, Yazd University, Yazd, Iran \\ ${ }^{2}$ Department of Physics, Faculty of Montazeri, Technical and Vocational University (TVU), Khorasan Razavi, Iran \\ ${ }^{3}$ Department of Physics, Hakim Sabzevari University, Sabzevar, Iran \\ ${ }^{4}$ International Centre for Theoretical Physics (ICTP), Associate and Federation Schemes, Medical Physics Field,
}

Trieste, Italy

\begin{abstract}
- Original article

*Corresponding authors:

M. Eslami-Kalantari, Ph.D.,

E-mail:meslami@yazd.ac.ir

Revised: February 2020

Accepted: March 2020

Int. J. Radiat. Res., October 2020; 18(4): 809-815

DOI: 10.18869 /acadpub.ijrr.18.4.809

Background: The total yields of direct Single-Strand Breaks (SSBs) and Double -Strand Breaks (DSBs) in proton energies varying from 0.1 to $40 \mathrm{MeV}$ were calculated. While other studies in this field have not used protons with energy less than $0.5 \mathrm{MeV}$, our results show interesting and complicated behavior of these protons. Materials and Methods: The simulation has been done using the Geant4-DNA toolkit. An atomic model of DNA geometry was simulated. Simulations were performed with a source in the Z-axis direction at the cell nucleus entrance with protons at energies of $0.1-1 \mathrm{MeV}$ in $0.1 \mathrm{MeV}$ steps, 5 $\mathrm{MeV}$, and $10-40 \mathrm{MeV}$ in $10 \mathrm{MeV}$ steps. Results: The calculated SSB yields decreased from 60.08 (GbpGy) ${ }^{-1}$ for $0.1 \mathrm{MeV}$ proton energy to 49.52 (GbpGy)

${ }^{-1}$ for $0.5 \mathrm{MeV}$ proton energy, and then it increased to 54.35 (GbpGy) ${ }^{-1}$ in 40 MeV. The DSB yields decreased from $4.32(\mathrm{GbpGy})^{-1}$ for $0.1 \mathrm{MeV}$ proton energy to 1.03 (GbpGy) $)^{-1}$ for $40-\mathrm{MeV}$ protons. The DSB yields for energies less than $0.5 \mathrm{MeV}$ was about $56 \%$, and for the other energy levels, it was $44 \%$. As for SSB yields, 35\% of the breaks arose from protons with an energy of fewer than $0.5 \mathrm{MeV}$ and $65 \%$ from higher energies. Conclusion: It was found that the proton ranges with an energy less than $0.5 \mathrm{MeV}$ are smaller than the cell size $(10 \mu \mathrm{m})$, and $100 \%$ of the energy is deposited in the cell region. Then protons with these energies are the best choice to increase the number of DSBs.
\end{abstract}

Keywords: Monte Carlo simulation, proton, SSB yield, DSB yield.

\section{INTRODUCTION}

Realizing radiation effects produced by charged particles through biological target is of great interest in treatment by radiation. Radiation therapy requires investigation of radiation cellular level effects and dose deposition originated from them on the nanoscale. The effects on human DNA by ionizing radiations are classified as direct and indirect damages. Direct damage is related to the direct action of particles (primary or secondary) on biological targets through ionization or excitation. Regarding strand breaks, DNA damages due to direct effects have been calculated and reported in different studies (1-5). In the exertion of indirect effects, the radiation interacts with water that fills most of the cell volume. This results in free radicals, which are highly reactive atoms or molecules, each with an unpaired electron. Free radicals can attack critical targets that consist of DNA and they also 
break their strands (6). Most DNA damages due to the direct or indirect effects involve Single Strand Breaks (SSBs) and Double - Strand Break (DSB). If two SSBs on opposite strands occur within ten base pairs, a DSB occurs (7-10). DSBs are considered as the most critical type of damage. They induce damages to chromosomes and lead to cell death. The DSB damage is more severe and may wrongly repair, which can result in cell death. The DNA molecule is a sensitive target to investigate biological effects regarding ionizing radiation in biological cells $(11,12)$. The development of a geometrical model is the first step that should be considered for modeling DNA damages. In the Monte Carlo simulation of DNA damages, some different configurations of DNA structures have been used. The models are of three different types, including linear cylinder, volumetric, and atomic models. The linear model is the simplest model that uses geometric cylindrical shapes to construct a DNA structure. In the volumetric model, the bases, sugar-phosphate groups, and the other parts of the DNA molecule are simulated using different shapes. A few of the researchers who reported their results based on this model include Humm and Charlton (13) and Nikjoo etal. (14). DNA strands are considered in detail in the atomic model, and atoms are simulated with spheres that are based on the Van der Waals radius of atoms. The concept of LET (Linear Energy Transfer) was introduced by the ICRU to compare the energy deposition events for different types of radiation (15). LET $(\mathrm{keV} / \mu \mathrm{m})$ is defined as the average energy transferred from radiation to a medium (for example tissues) per unit length of the path traveled by the radiation $(15,16)$. The radiation biological effects strongly depend on the LET, and that higher-LET radiation has a more significant effect on the cancer cell. One of the commonly used methods to study these effects in detail is based on Monte Carlo simulations performed by the track structure codes. Monte Carlo (MC) techniques are powerful tools for predicting the distribution of direct and indirect damages in cells due to ionizing radiation. Researchers use different simulation methods to study the radiation biological effects. There are different MC codes available to be used in simulations in the DNA size. They include the Geant4-DNA extension of the Geant4 toolkit (17-20), PENELOPE (21, 22), PARTRAC $(23,24)$, ETRACK $(25)$, CPA100 $(26,27)$, EPOTRAN (28), EGSnrc (29), and some others. The incident proton energy interval is $60-250 \mathrm{MeV}$, when these protons pass through different tissues, their energy lowers, and when they arrive in the cell nucleus, they will be so low energy. In the tissue and cell region, there are different range of energies, especially low energies. These low energy particles are important for destroying cancer cells. In this study, the Geant4-DNA toolkit was used to simulate protons with different delivered energies from $0.1 \mathrm{MeV}$ to $40 \mathrm{MeV}$ for calculating direct DNA damages. An atomic geometrical model was also defined based on Van der Waals radii. Then the number of SSBs, DSBs and the deposited doses were determined. The investigation proceeds with the calculation of the proton range in the cell region, LET values, SSB and DSB yields, and the Total Strand Break (TSB). The TSB is simply the sum of SSB and DSB yields (i.e., TSB $=$ SSB + DSB). The purpose of this study is to calculate breaks caused by different proton energies. As various studies have not investigated the breaks caused by protons below the $0.5 \mathrm{MeV}$, part of our study focused on this region of energy. In the end of the article, the effect of threshold energy values on SSB and DSB yields were shown.

\section{MATERIALS AND METHODS}

This section is organized into two parts. The first part explains the Geant4 Monte Carlo toolkit, and the second part introduces the simulated model of the cell nucleus and the methods of calculating DNA breaks.

\section{The Geant4 Monte Carlo toolkit}

The simulation in this study has been done using the Geant4-DNA toolkit for particle transport through the water. The GEANT4-DNA project is a new set of electromagnetic processes that serve to track low-energy electrons, protons, alpha particles, and several ions. The 
project is open-source and supported by many collaborators worldwide. The Geant4 toolkit, along with the Geant4-DNA extension, can transport protons in the energy interval of $1 \mathrm{keV}$ - $100 \mathrm{MeV}$ and electrons from $0.025 \mathrm{eV}$ to $1 \mathrm{MeV}$. The physical interactions between the incident protons and the DNA target were simulated with the G4EmDNAPhysics model, in which electromagnetic interactions are described. This model enables one to simulate specific physical processes such as elastic scattering, ionization, electronic excitation, and vibrational excitation for electrons. Interactions for proton beams are taken into consideration, too. The DNA materials are water because the available Geant4-DNA models use interaction cross sections in liquid water (30-32).

\section{Method of simulating the DNA geometry}

An atomic model of DNA was simulated using the Geant4 toolkit to calculate the total break yields. To construct the geometrical model of the cell nucleus, a technique was used to build different parts. The technique involved the simulation of nucleotide pairs as base pairs (bp) and the basic units to build up the whole genome structure, which is made up of 63 atoms, DNA double helix, nucleosome, and chromatin fibers. The properties of each atom, such as the corresponding chemical element, position, and nitrogen base, were simulated based on the geometry proposed by Bernal et al. (33). To form a DNA helix, base pairs were placed along a rotation axis at a turning angle of about $+36^{\circ}$ for each base pair. In the next step of the geometrical simulation, nucleosomes were constructed by turning two helical loops around a histone sphere. After the nucleosome construction, chromatin fibers were formed by six nucleosomes per level in a supra helix with a pitch of $7.11 \mathrm{~nm}$ and an external diameter of $31.38 \mathrm{~nm}$. The repetition of this structure led to a chromatin fiber. The DNA base pairs were all located randomly in a cell nucleus with a diameter of $10 \mu \mathrm{m}$. LET is defined as the amount of energy per unit length lost by a particle traversing a material based on equation 1 :

LET $=\frac{d E}{\mathrm{dx}}$
The range of particles in different energy levels and the deposited energy are calculated using the SRIM code and the Geant4 toolkit, respectively. Different types of damages, including base damage and strand breaks, were induced by radiation. The knowledge of the accurate position of strand breaks is necessary to realize the complexity of the damage. In this respect, two different groups of strand breaks, including SSB and DSB, were evaluated. At first, the SSBs, DSBs, and the deposited doses per event were determined. At the beginning of processing any events, it contains primary particles. These primaries are pushed into a stack. When the stack becomes empty, the processing of an event is over. G4Event class represents an event. Then DNA damage yields were calculated by equation 2 :

DSB (SSB) yield $=\frac{\text { Number of DSBs (SSBs) }}{\text { Dose (Gy) } \times \text { total number of base pairs (bp) }}$

The break yields were expressed in units of (Gy Gbp) ${ }^{-1}$. When energy deposition exceeds the threshold energy, an SSB occurs within the cell. The energy threshold in Geant4-DNA is the same as the first excitation value of water; it is $8.23 \mathrm{eV}$ in this study. Other energy threshold values, such as $10.79 \mathrm{eV}, 12.61 \mathrm{eV}$, and $17.5 \mathrm{eV}$ have been reported in other studies (21,34-37). The threshold energy was changed in this study to see its influence on the strand break yields. Simulations were performed with a source in the $\mathrm{Z}$-axis direction (perpendicular to the cell) at the cell nucleus entrance with mono-energetic protons hitting at energies of $0.1-1 \mathrm{MeV}$ in 0.1 $\mathrm{MeV}$ steps, $5 \mathrm{MeV}$, and 10-40 MeV in $10 \mathrm{MeV}$ steps. The number of primary particles used in this simulation for proton source included $10^{6}$ particles. We should emphasize that all outputs were presented in per source particle.

\section{RESULTS}

\section{SSB and DSB values for protons at different energy levels}

The number of DNA strand breaks (SSBs and DSBs) and the deposited dose per event, including their error bars (the error bars are so 
small to see, for example for the first point of SSB plot the value and error is $1.66 \pm 0.01$ ), are plotted as a function of energy in figure 1 .

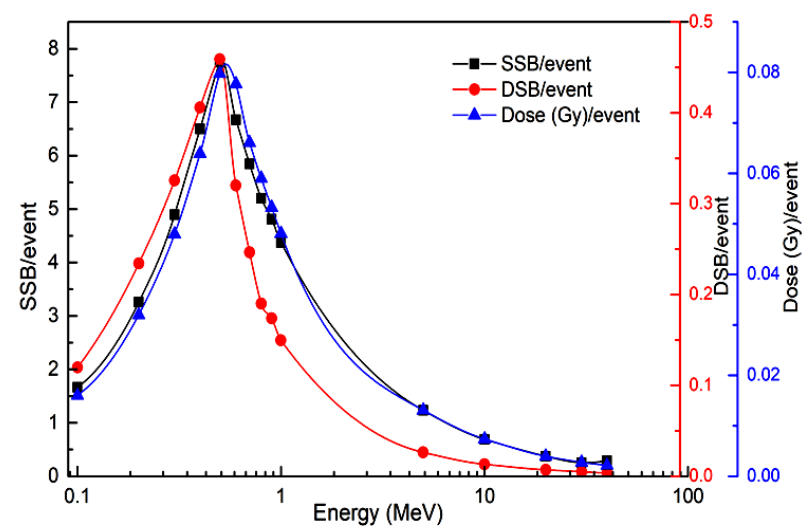

Figure 1. Number of the breaks and the deposited dose per event as a function of energy.

As figure 1 shows, the number of SSBs and DSBs is increased as the energy increases from $0.1 \mathrm{MeV}$ to $0.5 \mathrm{MeV}$. Beyond $0.5 \mathrm{MeV}$, with an increase in energy, the number of breaks is decreased. The trend of the deposited dose per event is similar to that of the SSBs and DSBs. It starts from about $0.02 \mathrm{~Gy}$ at $0.1 \mathrm{MeV}$ to the maximum value of $0.08 \mathrm{~Gy}$ at $0.5 \mathrm{MeV}$. After 0.5 $\mathrm{MeV}$, the dose decreases to $0.002 \mathrm{~Gy}$ at $40 \mathrm{MeV}$. To find the trend of the figures, we calculated the protons range for different energies by the SRIM code. Also, the energy deposit due to protons with different energies calculated by the Geant 4 toolkit. Table 1 shows the range of protons inside the cells, energy deposition, and the yields of both SSB and DSB as a function of proton energy. The SRIM code calculated the range of protons at different energy levels inside the cell nucleus.

As table 1 shows, for protons with the energy of less than $0.5 \mathrm{MeV}$, the range is less than the cell size $(10 \mu \mathrm{m})$. Thus, the total energy of the particles is deposited in the cell region. For energies more than $0.5 \mathrm{MeV}$, the range is bigger than the cell size, then the energy deposition reduces. It is interesting that, for protons with the energy of less than $0.5 \mathrm{MeV}$, the total proton energy is deposited inside the cells, whereas for $40-\mathrm{MeV}$ protons, the energy deposition is about 0.001 of the whole energy. The range of $40-\mathrm{MeV}$ protons is 1462 times bigger than the cell size, and most of the energy is deposited outside the cell region. For protons with the energy of less than $0.5 \mathrm{MeV}$, no calculation has been reported yet. Cell size has an important role in such a calculation. The LET values are calculated based on the results of table 1 by using equation 1 . Figure 2 show these calculations as well.

Table 1. The range of protons, energy deposition, and strand break yields as a function of proton energy.

\begin{tabular}{|c|c|c|c|c|c|}
\hline $\begin{array}{c}\text { Energy } \\
(\mathrm{MeV})\end{array}$ & $\begin{array}{c}\text { Range } \\
(\mu \mathrm{m})\end{array}$ & $\begin{array}{c}\text { Energy } \\
\text { deposition }(\mathrm{keV})\end{array}$ & $\begin{array}{c}\text { SSB } \\
(\text { GbpGy })^{-1}\end{array}$ & $\begin{array}{c}\text { DSB } \\
(\text { GbpGy })^{-1}\end{array}$ & $\begin{array}{c}\text { DSB/ } \\
\text { SSB }\end{array}$ \\
\hline 0.1 & 1.43 & 100.00 & 60.08 & 4.32 & 0.072 \\
\hline 0.2 & 2.79 & 199.94 & 58.74 & 4.22 & 0.072 \\
\hline 0.3 & 4.54 & 299.72 & 58.69 & 3.96 & 0.068 \\
\hline 0.4 & 6.68 & 399.38 & 58.67 & 3.66 & 0.064 \\
\hline 0.5 & 9.2 & 498.78 & 56.02 & 3.32 & 0.059 \\
\hline 0.6 & 12.07 & 485.72 & 49.52 & 2.38 & 0.048 \\
\hline 0.7 & 15.26 & 413.24 & 50.99 & 2.15 & 0.042 \\
\hline 0.8 & 18.27 & 368.70 & 51.99 & 1.98 & 0.039 \\
\hline 0.9 & 22.52 & 332.85 & 52.11 & 1.88 & 0.036 \\
\hline 1 & 26.55 & 300.40 & 52.53 & 1.79 & 0.034 \\
\hline 5 & 354.39 & 81.84 & 54.38 & 1.16 & 0.021 \\
\hline 10 & 1200 & 45.99 & 54.37 & 1.03 & 0.019 \\
\hline 20 & 4170 & 24.81 & 54.35 & 1.03 & 0.019 \\
\hline 30 & 8690 & 17.09 & 54.35 & 1.03 & 0.019 \\
\hline 40 & 14620 & 45.98 & 54.35 & 1.03 & 0.019 \\
\hline
\end{tabular}



Figure 2. LET for different proton energy levels.

The LET values for the protons with the energy interval of $0.1 \mathrm{MeV}-40 \mathrm{MeV}$ were found to be about $72.96-0.0031 \mathrm{keV} / \mu \mathrm{m}$. Figure 3 depicts the DSB yields and the DSB-to-TSB ratio as a function of the primary proton energy.

Int. J. Radiat. Res., Vol. 18 No. 4, October 2020 


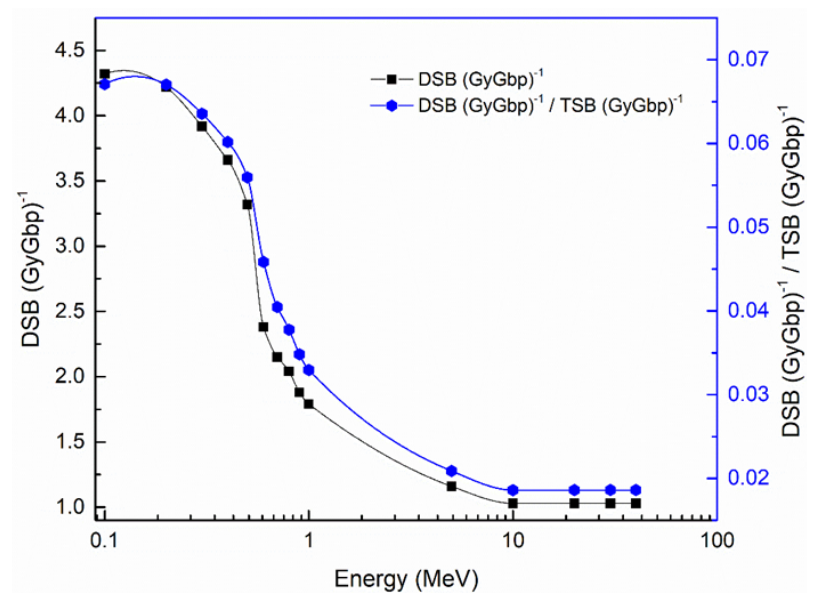

Figure 3. DSB yields as a function of energy and DSB-to-TSB ratio.

\section{The effect of threshold energy on DNA damages}

Table 2 shows the calculated break yields induced by mono-energetic $0.1 \mathrm{MeV}$ protons with different threshold energies often used in theoretical studies.

Table 2. Total calculated SSB and DSB yields corresponding to different threshold energies.

\begin{tabular}{|c|c|c|c|c|c|}
\hline $\begin{array}{c}\text { Threshold } \\
\text { energy } \\
(\mathbf{e V})\end{array}$ & $\begin{array}{c}\text { DSB (Gy } \\
\text { Gbp) }^{-1}\end{array}$ & $\begin{array}{c}\text { SSB (Gy } \\
\text { Gbp }^{-1}\end{array}$ & $\begin{array}{c}\text { DSB/ } \\
\text { DSB(8.23 } \\
\text { eV) }\end{array}$ & $\begin{array}{c}\text { SSB/ SSB } \\
(\mathbf{8 . 2 3} \text { eV) }\end{array}$ & $\begin{array}{c}\text { DSB / } \\
\text { TSB }\end{array}$ \\
\hline 8.23 & $4.32 \pm 0.21$ & $60.10 \pm 0.13$ & 1 & 1 & 0.07 \\
\hline 10.79 & $1.26 \pm 0.11$ & $29.12 \pm 0.09$ & 0.292 & 0.484 & 0.04 \\
\hline 12.61 & $0.73 \pm 0.09$ & $21.02 \pm 0.01$ & 0.169 & 0.349 & 0.03 \\
\hline 17.5 & $0.004 \pm 0001$ & $0.91 \pm 0.002$ & 0.001 & 0.015 & 0.01 \\
\hline
\end{tabular}

\section{DISCUSSION}

In this study, an atomic model of DNA was simulated, and the number of simple and complex damages, as well as SSB and DSB yields were calculated. The number of SSBs and DSBs and the amount of the dose increased in the energy interval of $0.1 \mathrm{MeV}-0.5 \mathrm{MeV}$. Beyond 0.5 $\mathrm{MeV}$, however, the trend reversed, and those values decreased. The ratio of the LET values of $0.1 \mathrm{MeV}$ to $0.5 \mathrm{MeV}$ was about 1.35 , and that for $40 \mathrm{MeV}$ was about 23163. These LET values were calculated based on the energy deposition and the range of protons in the cell nucleus.
Different studies have used different methods to calculate LET values. Some studies have calculated LET values at the center of the cell (38${ }^{40)}$ or in the cell entrance (41). Figure 2 shows that an increase in energy leads to a decrease in the LET. In general, as also found in other studies like $(42,43)$, Figure 3 suggests that DSB yields decrease once energy increases. The variation of DSB yields with proton energy is more than $100 \%$ (from 1.03 to 4.32 (Gy Gbp)-1). The experimental results obtained by Belli et al. showed the values 4.74 (Gy Gbp)-1 for $11 \mathrm{keV} /$ $\mu \mathrm{m}$ and 5.77 (Gy Gbp) ${ }^{-1}$ for $31 \mathrm{keV} / \mu \mathrm{m}{ }^{(44)}$. Our results for these two LETs are about 50\% lower than Belli results. This difference comes from the including of indirect breaks in their calculations that we did exclude them in our calculations. It is important to emphasize that the researchers only calculated direct damages, and their results did not address indirect ones. The ratio of breaks from direct and indirect effects, as reported in some studies, is about 35/65 for the cobalt source (45). The ratio of DSB yields from the proton energy of $0.1 \mathrm{MeV}$ to $0.5 \mathrm{MeV}$ is about 1.3, whereas it is about 4.19 for $0.1 \mathrm{MeV}$ and $40 \mathrm{MeV}$ protons. For higher energy levels, DSBs go to a constant value because most of their energy is deposited outside the cell region. DSB yields at $0.1 \mathrm{MeV}$ are about 4.2 times bigger than those at $40 \mathrm{MeV}$. Low energies, especially those less than $0.5 \mathrm{MeV}$, account for the majority of damages. The ratio of DSB to TSB varies from 0.065 (at 0.1 $\mathrm{MeV}$ ) to 0.02 (at $40 \mathrm{MeV}$ ). As found in other studies such as (36), figure 4 suggests that for energies higher than $0.5 \mathrm{MeV}$, the SSB yields increase when the energy is increased. For proton energies less than $0.5 \mathrm{MeV}$, the trend is interesting, which is ignored by other studies. SSB yields at $0.5 \mathrm{MeV}$ lose about $18 \%$ of their value at $0.1 \mathrm{MeV}$, but the trend changes beyond this energy; they increase for about $9 \%$ in comparison with their value at $0.5 \mathrm{MeV}$. Direct SSB and DSB yields seem to be constant beyond $5 \mathrm{MeV}$. Based on figure 4, most of the breaks are of the SSB type, and they vary from about 0.93 (at $0.1 \mathrm{MeV}$ ) to 0.98 (at $40 \mathrm{MeV}$ ) of the total strand breaks. The SSB-to-TSB ratio increases with an increase in energy. The last results were related to the different threshold energy to see 
the effect of this value on break yields. The total yields of SSB and DSB are decreased when the threshold energy is increased. The decreasing rate of DSB is faster than that of SSB; the ratio of DSB at the threshold energy of $10.79 \mathrm{eV}$ to that at the threshold energy of $8.23 \mathrm{eV}$ is about $\% 29$. For SSB, this ratio is about \%48. The ratios of DSBs at $10.79 \mathrm{eV}, 12.61 \mathrm{eV}$, and $17.5 \mathrm{eV}$ to 8.23 $\mathrm{eV}$ were $0.29,0.17$, and 0.001 respectively. For SSB yields, the corresponding ratios were 0.48 , 0.35 , and 0.015 . It shows that different studies should consider this value, and its effect on the break yields is so much.

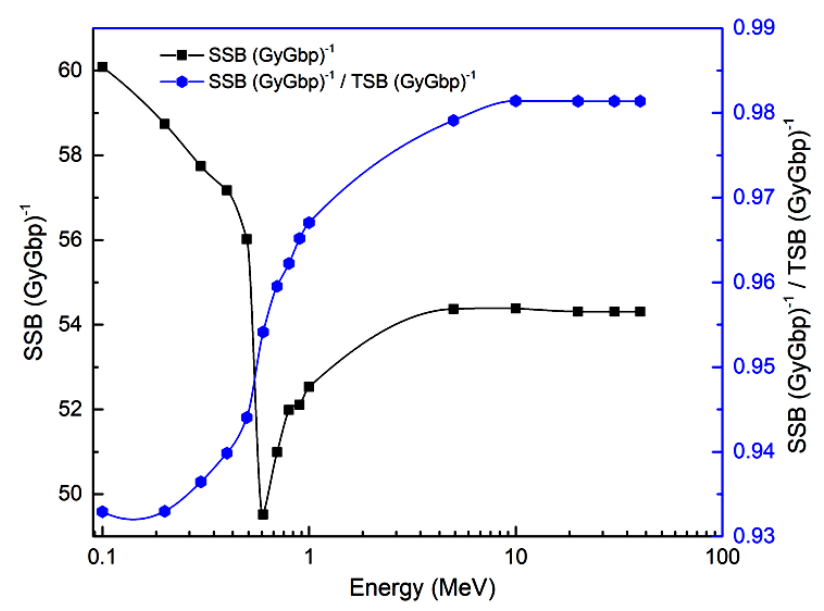

Figure 4. Displays the SSB yields as well as the SSB-to-TSB ratio as a function of proton energy.

\section{CONCLUSION}

In this study, Geant4-DNA simulations were done to evaluate the direct damages caused by primary protons in the energy interval of 0.1-40 $\mathrm{MeV}$. The trend of DSB yields decreased from 4.32 to 1.03 (GbpGy) $^{-1}$ in the energy interval of $0.1 \mathrm{MeV}$ to $40 \mathrm{MeV}$. The situation was different for the SSB yields. At energy levels lower than $0.5 \mathrm{MeV}$, the SSB yields decreased from 60.08 (GbpGy) $^{-1}$ to 49.52 (GbpGy) $^{-1}$ and Beyond that the yields was increased. Therefore, as the LET increased, for energy less than $0.5 \mathrm{MeV}$, the proton ranges were bigger than the cell size. The absolute yields of the SSBs tended to decrease, whereas that of the DSBs increased. Generally speaking, the cell size has an important role in calculations.

\section{4}

Funding: There is no fund for this study.

Conflicts of interest: Declared none.

\section{REFERENCES}

1. Friedland $W$, Jacob $P$, Paretzke HG, Merzagora M, Ottolenghi A (1999) Simulation of DNA fragment distributions after irradiation with photons. Radiat Environ Biophys, 38 (1): 39-47.

2. Yokoya A, Shikazono N, Fujii K, Urushibara A, Akamatsu K, Watanabe $R$ (2008) DNA damage induced by the direct effect of radiation. Radiat Phys Chem, 77(10): 1280-5.

3. Shao C, Saito M, Yu Z (1999) Formation of single- and double-strand breaks of pBR322 plasmid irradiated in the presence of scavengers. Radiat Environ Biophys, 38(2): 105-9.

4. Milian FM, Gouveia AN, Gual MR, Echeimberg JO, ArrudaNeto JDT, Garcia F, et al. (2007) In Vitro Effects of Gamma Radiation from 60Co and 137Cs on Plasmid DNA. J Biol Phys, 33(2): 155-60.

5. Hosseini MA, Jia SB, Ebrahimi-Loushab M (2017) Analysis of Relative Biological Effectiveness of proton beams and iso-effective dose profiles using Geant4. J Biomed Phys Eng, 7(2): 95-100.

6. Elgazzar AH and Kazem N (2006) Biological Effects of lonizing Radiation. In: Elgazzar AH, editor. The Pathophysiologic Basis of Nuclear Medicine. Berlin, Heidelberg: Springer Berlin Heidelberg, p. 540-8.

7. Lampe N, Karamitros M, Breton V, Brown JMC, Kyriakou I, Sakata D, et al. (2018) Mechanistic DNA damage simulations in Geant4-DNA part 1: A parameter study in a simplified geometry. Phys Med, 48: 135-45.

8. Tajik M, Rozatian ASH, Semsarha F (2015) Simulation of ultrasoft X-rays induced DNA damage using the Geant4 Monte Carlo toolkit. Instrum Methods Phys Res B, 342: 258-65.

9. Nikjoo H, O'Neill P, Goodhead DT, Terrissol M (1997) Computational modelling of low-energy electron-induced DNA damage by early physical and chemical events. Int J Radiat Biol, 71(5): 467-83.

10. Nikjoo $H$, Taleei $R$, Liamsuwan $T$, Liljequist $D$, Emfietzoglou D (2016) Perspectives in radiation biophysics: From radiation track structure simulation to mechanistic models of DNA damage and repair. Radiat Phys Chem, 128: 3-10.

11. Friedland $W$, Jacob $P$, Kundrát $P$ (2011) Mechanistic simulation of radiation damage to DNA and its repair: on the track towards systems radiation biology modelling. Radiat Prot Dosimetry, 143(2-4): 542-8.

12. Friedland $W$ and Kundrát $P$ (2013) Track structure based modelling of chromosome aberrations after photon and alpha-particle irradiation. Mutat Res Genet Toxicol Environ Mutagen, 756(1): 213-23.

13. Charlton DE, Nikjoo H, Humm JL (1989) Calculation of Initial Yields of Single- and Double-strand Breaks in Cell Nu-

Int. J. Radiat. Res., Vol. 18 No. 4, October 2020 
clei from Electrons, Protons and Alpha Particles. Int J Radiat Biol, 56(1):1-19.

14. Nikjoo H, O'Neill P, Terrissol M, Goodhead DT (1999) Quantitative modelling of DNA damage using Monte Carlo track structure method. Radiat Environ Biophys, 38(1): 31 8.

15. Report 16 (2016). J ICRU, os9(1): NP-NP.

16. Report 85 (2011) Fundamental quantities and units for ionizing radiation. J ICRU, 11(1): 1-31.

17. Francis Z, Incerti S, Capra R, Mascialino B, Montarou G, Stepan V, et al. (2011) Molecular scale track structure simulations in liquid water using the Geant4-DNA MonteCarlo processes. Appl Radiat Isot, 69(1): 220-6.

18. Incerti S, Baldacchino G, Bernal M, Capra R, Champion C, Francis Z, et al. (2009) The Geant4-DNA Project. Int J Mod Simul Scient Comput, 01(02):157-78.

19. Muñoz A, Pérez JM, García G, Blanco F (2010) An approach to Monte Carlo simulation of low-energy electron and photon interactions in air. Instrum Methods Phys Res $A$, 536(1): 176-88.

20. Allison J, Amako K, Apostolakis J, Arce P, Asai M, Aso T, et al. (2016) Recent developments in Geant4. Instrum Methods Phys Res A, 835: 186-225.

21. Bernal MA and Liendo JA (2009) An investigation on the capabilities of the PENELOPE MC code in nanodosimetry. Med Phys, 36(2): 620-5.

22. Baró J, Sempau J, Fernández-Varea JM, Salvat F (1995) PENELOPE: An algorithm for Monte Carlo simulation of the penetration and energy loss of electrons and positrons in matter. Instrum Methods Phys Res B, 100(1): 31-46.

23. Friedland W, Dingfelder $M$, Kundrat $P$, Jacob $P$ (2011) Track structures, DNA targets and radiation effects in the biophysical Monte Carlo simulation code PARTRAC. Mutat Res Genet Toxicol Environ Mutagen, 711(1-2):28-40.

24. Friedland W, Dingfelder M, Jacob P, Paretzke HG (2005) Calculated DNA double-strand break and fragmentation yields after irradiation with He ions. Radiat Phys Chem, 72 (2): 279-86.

25. Ito A (1988) Electron Track Simulation For Microdosimetry. In: Jenkins TM, Nelson WR, Rindi A, editors. Monte Carlo Transport of Electrons and Photons. Boston, MA: Springer US, p. 361-82.

26. Terrissol M, Edel S, Pomplun E (2004) Computer evaluation of direct and indirect damage induced by free and DNA-bound iodine-125 in the chromatin fibre. Int J Radiat Biol, 80(11-12): 905-8.

27. Terrissol M and Beaudré A (1990) Simulation of Space and Time Evolution of Radiolytic Species Induced by Electrons in Water. Radiat Prot Dosimetry, 31(1-4): 175-7.

28. Champion C, Le Loirec C, Stosic B (2012) EPOTRAN: A fulldifferential Monte Carlo code for electron and positron transport in liquid and gaseous water. Int J Radiat Biol, $8 \mathbf{8}$ (1-2): 54-61.

29. Kawrakow I (2000) Accurate condensed history Monte Carlo simulation of electron transport. I. EGSnrc, the new EGS4 version. Med Phys, 27(3): 485-98.

30. Incerti S, Douglass M, Penfold S, Guatelli S, Bezak E (2016) Review of Geant4-DNA applications for micro and na- noscale simulations. Phys Med, 32(10): 1187-200.

31. Incerti S (2014) The geant4-DNA project: overview and status. Phys Med, 30: e8.

32. Chauvie S, Francis Z, Guatelli S, Incerti S, Mascialino B, Moretto P, et al. (2007) Geant4 Physics Processes for Microdosimetry Simulation: Design Foundation and Implementation of the First Set of Models. IEEE Trans Nucl Sci, 54(6): 2619-28.

33. Bernal MA, Sikansi D, Cavalcante F, Incerti S, Champion C, Ivanchenko V, et al. (2013) An atomistic geometrical model of the B-DNA configuration for DNA-radiation interaction simulations. Comput Phys Commun, 184(12): 2840-7.

34. Friedland $W$, Jacob $P$, Paretzke HG, Merzagora M, Ottolenghi A (1999) Simulation of DNA fragment distributions after irradiation with photons. Radiat Environ Biophys, 38 (1): 39-47.

35. Goorley T, Terrissol M, Nikjoo H (2008) Calculated strand breaks from 1251 in coiled DNA. Int J Radiat Biol, 84(12): 1050-6.

36. Pomplun E (1991) A new DNA target model for track structure calculations and its first application to I-125 Auger electrons. Int J Radiat Biol, 59(3): 625-42.

37. Delage E, Pham QT, Karamitros M, Payno H, Stepan V, Incerti S, et al. (2015) PDB4DNA: Implementation of DNA geometry from the Protein Data Bank (PDB) description for Geant4-DNA Monte-Carlo simulations. Comput Phys Commun, 192: 282-8.

38. Bernal MA, deAlmeida CE, Sampaio C, Incerti S, Champion C, Nieminen $P$ (2011) The invariance of the total direct DNA strand break yield. Med Phys, 38(7):4147-53.

39. Villagrasa C, Meylan S, Gonon G, Gruel G, Giesen U, Bueno $M$, et al.(2017) Geant4-DNA simulation of DNA damage caused by direct and indirect radiation effects and comparison with biological data. Epj Web Conf, 153: 04019.

40. Shamshiri P, Forozani G, Zabihi A (2019) An investigation of the physics mechanism based on DNA damage produced by protons and alpha particles in a realistic DNA model. Instrum Methods Phys Res B, 454: 40-4.

41. Campa A, Ballarini F, Belli M, Cherubini R, Dini V, Esposito $G$, et al. (2005) DNA DSB induced in human cells by charged particles and gamma rays: experimental results and theoretical approaches. Int J Radiat Biol, 81(11): 84154.

42. Mokari M, Alamatsaz MH, Moeini H, Taleei R (2018) A simulation approach for determining the spectrum of DNA damage induced by protons. Phys Med Biol, 63 (17):175003.

43. Meylan S, Incerti S, Karamitros M, Tang N, Bueno M, Clairand I, et al.( 2017) Simulation of early DNA damage after the irradiation of a fibroblast cell nucleus using Geant4DNA. Sci Rep, 7(1): 11923.

44. Belli M, Cherubini R, Dalla Vecchia M, Dini V, Moschini G, Signoretti C, et al. (2000) DNA DSB induction and rejoining in V79 cells irradiated with light ions: a constant field gel electrophoresis study. Int J Radiat Biol, 76(8): 1095-104.

45. Friedland $W$, Jacob $P$, Bernhardt $P$, Paretzke HG, Dingfelder M (2003) Simulation of DNA damage after proton irradiation. Radiat Res, 59(3): 401-10. 
